\title{
Carcinoma de células en anillo de sello gástrico: Descripción clínico-morfológica y valor pronóstico*
}

\author{
Drs. OSCAR TAPIA E. ${ }^{1,2}$, VALENTINA GUTIÉRREZ M. ${ }^{1}$, JUAN CARLOS ROA S. ${ }^{1,2}$, \\ CARLOS MANTEROLA D. ${ }^{3}$, MIGUEL VILLASECA H. ${ }^{1,2}$, JUAN CARLOS ARAYA O.$^{1,2}$ \\ 1 Departamento de Anatomía Patológica. \\ 2 Scientific and Technological Bioresource Nucleus (BIOREN). \\ 3 Departamento de Cirugía y Traumatología, Facultad de Medicina. Universidad de La Frontera. \\ Temuco, Chile.
}

\begin{abstract}
Prognosis of signet ring cell carcinoma of the stomach

Background: Signet ring cell carcinoma of the stomach corresponds to 3 to $39 \%$ of all gastric malignant tumors, and its prognostic significance is not well known. Aim: To compare the prognosis of signet ring cell carcinoma of the stomach with other types of gastric cancer. Material and Methods: Review of 451 patients operated for gastric carcinoma. Signet ring cell carcinomas were compared with the rest of tumors. Results: One hundred twenty tumors $(27 \%)$ were signet ring cell carcinoma, they were more commonly located in the lower third of the stomach, were less differentiated, bigger and had more lymph nodes involved. Patients with this variety of tumor were younger, more often women and their survival was lower, even when separating incipient and advanced tumors. Conclusions: Signet ring cell carcinoma of the stomach had a worst prognosis that other type of gastric tumors.
\end{abstract}

Key words: Gastric neoplasm, signet ring cell carcinoma, prognosis.

\section{Resumen}

Introducción: La incidencia de carcinoma gástrico (CG) de células en anillo de sello (CAS) varía de $3 \%$ a $39 \%$. Trabajos de supervivencia en pacientes con tumores gástricos reportan resultados contradictorios en cuanto a la importancia pronóstica del tipo histológico CAS respecto a los otros tipos histológicos. El objetivo del estudio es describir y evaluar la importancia pronóstica del tipo histológico CAS comparado con los carcinomas no anillo de sello (CNAS). Material y Método: Estudio de cohorte retrospectiva. Se estudiaron variables clínicas y morfológicas de 451 pacientes operados por CG en el Hospital Hernán Henríquez Aravena de Temuco entre Enero/1986-Diciembre/2001 agrupándose para el análisis según el diagnóstico histopatológico en CAS y CNAS. Se realizó un análisis exploratorio de los datos y posteriormente se aplicó estadística descriptiva con cálculo de medidas de tendencia central y extrema; y estadísticas analíticas, aplicando Chi cuadrado de Pearson y test exacto de Fisher para variables categóricas, T-Student para variables continuas,

*Recibido el 5 de Marzo de 2010 y aceptado para publicación el 20 de Abril de 2010.

Financiado en parte por la Dirección de Investigación de la Universidad de La Frontera.

Correspondencia: Dr. Oscar Tapia E.

Manuel Montt 112. Código Postal 478-1176. Temuco, Chile.

E-mail: otescalona@gmail.com 
Kaplan-Meier y Log-rank test para análisis de supervivencia. Resultados: Un 27\% (120) correspondió a CAS observándose diferencias estadísticamente significativas entre los grupos CAS y CNAS para las siguientes variables: género, edad, localización y tamaño tumoral, grado de diferenciación histológico y compromiso ganglionar linfático. El análisis de supervivencia demostró un peor pronóstico para el grupo de CAS ( $p=$ 0,02). En el análisis por separado de tumores incipientes y avanzados respecto del tipo histológico, no observamos diferencias significativas para lesiones incipientes $(\mathrm{p}=0,07)$ mientras que para lesiones avanzadas el CAS se asocia a un pronóstico desfavorable $(\mathrm{p}<0,0001)$. Conclusiones: Observamos en nuestra serie un peor pronóstico asociado al tipo histológico CAS respecto a los otros tipos histológicos (CNAS), información crucial que, aportada al clínico, deberá ser considerada al momento de decidir terapias para perfiles tumorales específicos.

Palabras clave: Neoplasia gástrica, carcinoma de células en anillo de sello, pronóstico.

\section{Introducción}

El cáncer gástrico $(\mathrm{CG})$ sigue siendo la primera causa de muerte por cáncer en Chile, con una tasa de mortalidad general de $19,5 \times 10^{5}$ habitantes y de $25,3 \times 10^{5}$ en hombres; en la IX Región la tasa de mortalidad general es de $24,8 \times 10^{5}$ habitantes; y en hombres de $33,7 \times 10^{5}$. . Si bien en las últimas 3 décadas se ha observado una estabilización en la tasa de mortalidad general por $\mathrm{CG}$, hoy en día la probabilidad media de que un chileno muera por CG es de aproximadamente $3 \%$, razón por la que el CG es aún problema de salud pública ${ }^{1,5,6}$.

El análisis de la pieza operatoria ha permitido identificar variables anatomo-patológicas con valor pronóstico en la supervivencia y recurrencia de la enfermedad en estos pacientes, siendo el compromiso ganglionar linfático $(\mathrm{N})$ y nivel de infiltración tumoral en la pared gástrica $(\mathrm{T})$ de los factores más relevantes identificados. En distintos estudios se mencionan otros factores morfológicos pronósticos, como tamaño, localización y forma tumoral, grado de diferenciación histológica, tipo histológico (anillo de sello y no anillo de sello), barrera ganglionar linfática comprometida, metástasis a distancia, estadio TNM y presencia de tumor residual ${ }^{6-19}$.

En cuanto al tipo histológico, los carcinomas gástricos se pueden agrupar de la siguiente forma: carcinoma anillo de sello (CAS) y carcinoma no anillo de sello (CNAS) incluyéndose en este último grupo los carcinomas tubular, papilar, mucinoso y otras variedades menos frecuentes tales como adenoescamoso y escamoso puro. El CAS gástrico se origina en mucosa sin metaplasia a nivel de la zona proliferativa del cuello de las glándulas y, de acuerdo a la clasificación de la World Health Organization (WHO) corresponde a aquel tumor examinado en la pieza quirúrgica donde más del $50 \%$ de las células neoplásicas son células en anillo de sello ${ }^{20-23}$. Esta variedad histológica representa entre el 3-39\% de los carcinomas gástricos, siendo también clasificado como tipo difuso por Lauren o infiltrativo por Ming, caracterizándose por afectar típicamente a sujetos más jóvenes y en una mayor proporción mujeres comparado con los CNAS. Desde el punto de vista morfológico, los CAS son tumores infiltrativos que tienden a comprometer todo el espesor de la pared gástrica y presentar frecuente compromiso tumoral ganglionar linfático al momento del diagnóstico asociándose por tanto a un peor pronóstico ${ }^{21,22,24}$.

Los trabajos publicados reportan resultados contradictorios en cuanto a la importancia pronóstica del tipo histológico anillo de sello en tumores gástricos, donde algunos han demostrado un peor pronóstico para el CAS, otros no demuestran diferencias estadísticas y por el contrario, otros reportan un mejor pronóstico para el CAS respecto al CNAS. Junto a estos dispares resultados, autores atribuyen a su vez distinta importancia pronóstica del tipo histológico según se trate de un cáncer gástrico incipiente o avanzado; en el primer caso tendrían mejor supervivencia a 5 años que los CNAS mientras que en tumores avanzados los resultados demuestran una peor supervivencia en los pacientes con $\mathrm{CAS}^{24-28}$.

El objetivo de este estudio es describir variables clínico-morfológicas para los tumores gástricos tipo CAS y CNAS junto con valorar el impacto en la supervivencia del tipo histológico CAS al compararlos con los CNAS.

\section{Material y Método}

Diseño del estudio: Cohorte histórica.

Marco: Se recogieron los datos provenientes de pacientes diagnosticados de CG en la Unidad de Anatomía Patológica del Hospital Hernán Henríquez Aravena de Temuco entre enero de 1986 y diciembre de 2001 ( $\mathrm{n}=451)$.

Participantes: Se incluyeron todos los casos de gastrectomías por CG diagnosticados de forma consecutiva en el período e institución antes señalados y en los cuales se realizó tratamiento quirúrgico con disección ganglionar extendida (D2). El seguimiento 
de los casos se realizó mediante el estudio de las historias clínicas y certificados de defunción obtenidos del Registro Civil e Identificación.

Criterios de exclusión: Fueron excluidos aquellos sujetos sin indicación quirúrgica por enfermedad avanzada o condiciones clínicas del paciente, casos sometidos sólo a laparotomía exploradora por enfermedad diseminada y pacientes sometidos a cirugías paliativas. Se excluyeron del análisis de supervivencia pacientes que fallecieron en los 30 días posteriores a la cirugía, considerados como mortalidad perioperatoria (16 casos).

Protocolo de procesamiento. En este período, el procesamiento de las piezas quirúrgicas de gastrectomía fue realizado en forma uniforme a través de la apertura, extensión y fijación de la pieza quirúrgica en planchas de parafina sólida, con un examen macroscópico diferido. El examen microscópico consideró el estudio del tumor, ganglios linfáticos $\mathrm{y}$ ambos bordes de sección quirúrgicos (proximal y distal). El tumor fue examinado mediante múltiples secciones con el objeto de determinar el máximo nivel de infiltración en la pared gástrica y representatividad histológica de la lesión tumoral.

Variables de interés: Para ambos tipos de tumores (CAS y CNAS) se describen variables clínicas obtenidas de las fichas (género, edad y estadio TNM) y morfológicas obtenidas del informe anatomo-patológico (localización, tamaño tumoral, nivel de infiltración, grado de diferenciación histológico y compromiso ganglionar linfático). Finalmente se comparó la supervivencia global para ambos grupos de tumores y en forma independiente para tumores incipientes y avanzados según el tipo histológico.

Plan de análisis: Se realizó un análisis exploratorio de los datos, lo que determinó la necesidad de categorizar algunas variables según su distribución, como tamaño tumoral ( $<60 \mathrm{~mm}$ y $\geq 60 \mathrm{~mm}$ ), localización tumoral (categorizada en tres tercios: superior [cardial y subcardial], medio [cuerpo] e inferior [antral y pilórico]) acorde con las normas de la sociedad japonesa (en aquellos casos de tumores extensos con compromiso de más de un segmento, se definió según la ubicación del centro de la lesión), nivel de infiltración (categorizado según el compromiso de la pared gástrica en tumores incipientes [infiltran mucosa o submucosa] y tumores avanzados [infiltran túnica muscular propia, subserosa o serosa]), grado de diferenciación histológica (categorizado en bien diferenciado, moderadamente diferenciado y poco diferenciado), prevaleciendo para su categorización el menor grado de diferenciación presente en el tumor, compromiso ganglionar linfático y estadio TNM (de acuerdo a la definición propuesta por la AJCC).

Sesgos: Se minimizó el sesgo de clasificación mediante la comprobación de los datos por investigadores independientes con amplia experiencia en el tema (JCR, MV, JCA y PG).

Tamaño de la muestra: No se realizó estimación de tamaño de la muestra pues se trabajó con la totalidad del universo de pacientes con CG sometidos a gastrectomía en el período señalado.

Métodos estadísticos: Utilizando los paquetes estadísticos Epi-info 6.0 y Stata 9.0, se realizó un análisis exploratorio de los datos y posteriormente se aplicó estadística descriptiva con cálculo de medidas de tendencia central y extrema; y estadísticas analíticas, aplicando Chi cuadrado de Pearson y test exacto de Fisher para variables categóricas, T-Student para variables continuas, Kaplan-Meier y Log-rank test para análisis de supervivencia.

\section{Resultados}

En la cohorte analizada el 27\% (120) de los casos correspondió a CAS y 73\% (331) a CNAS, comparándose las características clínico-patológicas de ambos grupos en la Tabla 1. Se observaron diferencias estadísticamente significativas entre el tipo CAS y CNAS para las siguientes variables: género, edad, localización y tamaño tumoral, grado de diferenciación histológico y compromiso ganglionar linfático, donde los pacientes con CAS son más jóvenes, en una mayor proporción mujeres que presentan tumores poco diferenciados de mayor tamaño y con frecuente compromiso linfonodal. No observamos diferencias estadísticas significativas en cuanto al nivel de infiltración ni estadio TNM.

La supervivencia global a 5 años de pacientes con CAS y CNAS fue $37 \%$ y $51 \%$ respectivamente $(\mathrm{p}=$ 0,02) (Figura 1).

Para el grupo de tumores incipientes, la supervivencia global a 5 años para los pacientes con CAS y CNAS fue de $95 \%$ y $84 \%$ respectivamente $(\mathrm{p}=$ 0,07) (Figura 2).

Para el grupo de tumores avanzados, la supervivencia global a 5 años para los pacientes con CAS y CNAS fue de $26 \%$ y $45 \%$ respectivamente ( $\mathrm{p}<$ 0,001) (Figura 3).

\section{Discusión}

La incidencia de CAS gástrico reportada varía desde 3,4\% en Japón a 39\% en países occidentales; en nuestra serie el $27 \%$ correspondió a esta variedad histológica, frecuencia similar a lo reportado por Antonioli et al, quienes encontraron hasta un 29\% de pacientes con carcinoma gástrico de células en anillo de sello en la serie estudiada ${ }^{25,29,30}$. 
CARCINOMA DE CÉLULAS EN ANILLO DE SELLO GÁSTRICO: DESCRIPCIÓN CLÍNICO-MORFOLÓGICA Y VALOR ...

Tabla 1. Distribución de variables clínico-morfológicas en pacientes con CAS y CNAS $(\mathrm{n}=451)$

\begin{tabular}{|c|c|c|c|}
\hline \multirow[b]{2}{*}{ Variables } & \multicolumn{3}{|c|}{ Cáncer Gástrico } \\
\hline & $\begin{array}{c}\text { Carcinoma de Células en anillo } \\
\text { de sello (CAS) } \\
\%(n=120)\end{array}$ & $\begin{array}{c}\text { Otros Tipos Histológicos de } \\
\text { Carcinoma (CNAS) } \\
\%(n=331)\end{array}$ & $\mathbf{p}$ \\
\hline Género & & & $<0,001$ \\
\hline Femenino & $46,0(55)$ & $29,0(95)$ & \\
\hline Masculino & $54,0(65)$ & $71,0(236)$ & \\
\hline Edad (promedio) & $55,5 \pm 13,3$ años & $61,8 \pm 10,6$ años & $<0,001$ \\
\hline Nivel de infiltración & & & 0,66 \\
\hline Incipiente & $16,0(19)$ & $14,0(47)$ & \\
\hline Avanzado & $84,0(101)$ & $86,0(284)$ & \\
\hline Localización tumoral & & & $<0,001$ \\
\hline $1 / 3$ Superior & $16,0(19)$ & $31,0(102)$ & \\
\hline 1/3 Medio & $27,0(32)$ & $30,0(99)$ & \\
\hline $1 / 3$ Inferior & $50,0(61)$ & $38,0(126)$ & \\
\hline Linitis & $7,0 \quad(8)$ & 1,0 & \\
\hline Grado de diferenciación & & & $<0,001$ \\
\hline Bien & - & $11,0(36)$ & \\
\hline Moderado & $4,0 \quad(5)$ & $41,0(137)$ & \\
\hline Poco & $96,0(115)$ & $48,0(158)$ & \\
\hline Tamaño tumoral & & & 0,04 \\
\hline$<60 \mathrm{~mm}$ & $43,0(52)$ & $54,0(178)$ & \\
\hline$\geq 60 \mathrm{~mm}$ & $57,0(68)$ & $46,0(153)$ & \\
\hline Tamaño tumoral (promedio) & $65,8 \pm 35,4 \mathrm{~mm}$ & $61,8 \pm 32,1 \mathrm{~mm}$ & 0,13 \\
\hline Infiltración & & & 0,19 \\
\hline $\mathrm{T} 1$ & $12,0(14)$ & $15,0(49)$ & \\
\hline $\mathrm{T} 2$ & $40,0(48)$ & $45,0(150)$ & \\
\hline $\mathrm{T} 3$ & $44,0(53)$ & $38,0(127)$ & \\
\hline $\mathrm{T} 4$ & $4,0 \quad(5)$ & $2,0 \quad(5)$ & \\
\hline Ganglios resecados (promedio) & $31,9 \pm 18,4$ & $31,2 \pm 16,7$ & 0,92 \\
\hline Compromiso ganglionar & & & 0,03 \\
\hline Sin compromiso & $25,0(30)$ & $36,0(118)$ & \\
\hline Con compromiso & $75,0(90)$ & $64,0(213)$ & \\
\hline Ganglios positivos (promedio) & 9,38 & 5,48 & $<0,001$ \\
\hline Estado Ganglionar & & & 0,01 \\
\hline N0 & $21,0(25)$ & $33,0(110)$ & \\
\hline N1 & $38,0(46)$ & $37,0(123)$ & \\
\hline N2 & $40,0(48)$ & $30,0(98)$ & \\
\hline N3 & $1,0 \quad(1)$ & - & \\
\hline Estadio TNM & & & 0,22 \\
\hline I & $22,0(27)$ & $27,0(90)$ & \\
\hline II & $23,0(28)$ & $26,0(87)$ & \\
\hline III & $45,0(53)$ & $42,0(137)$ & \\
\hline IV & $10,0(12)$ & 5,0 & \\
\hline Supervivencia (promedio) & 45,5 meses & 54,4 meses & 0,04 \\
\hline
\end{tabular}




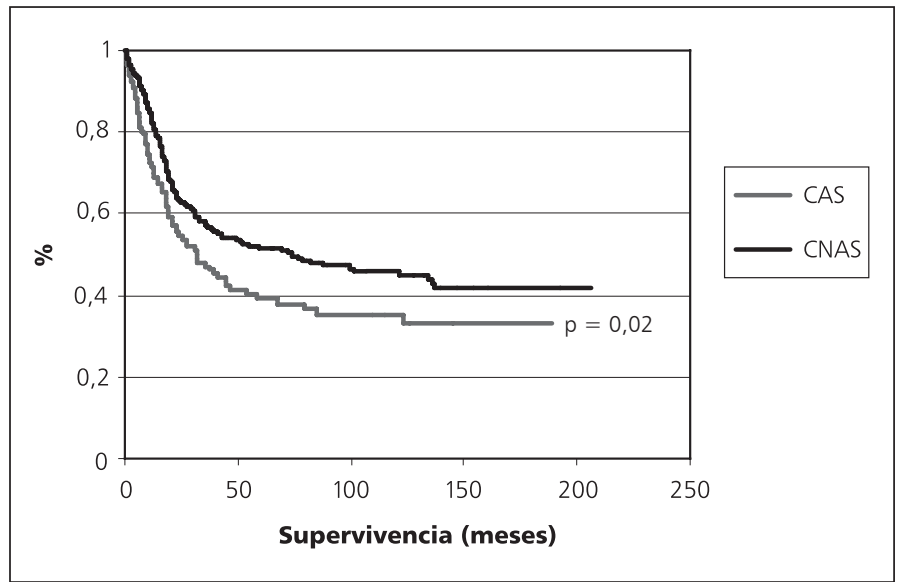

Figura 1. Supervivencia global según tipo histológico.

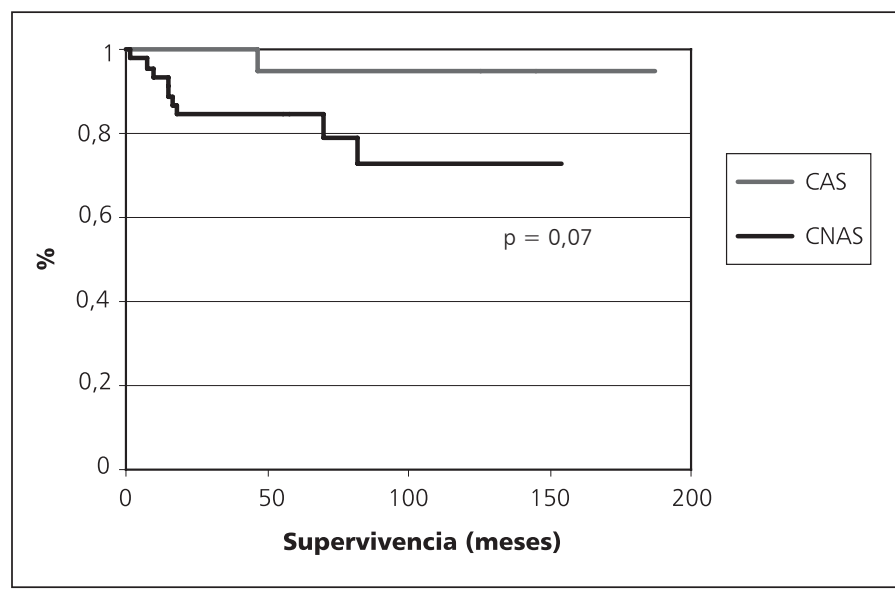

Figura 2. Supervivencia según tipo histológico para tumores gástrico incipientes.

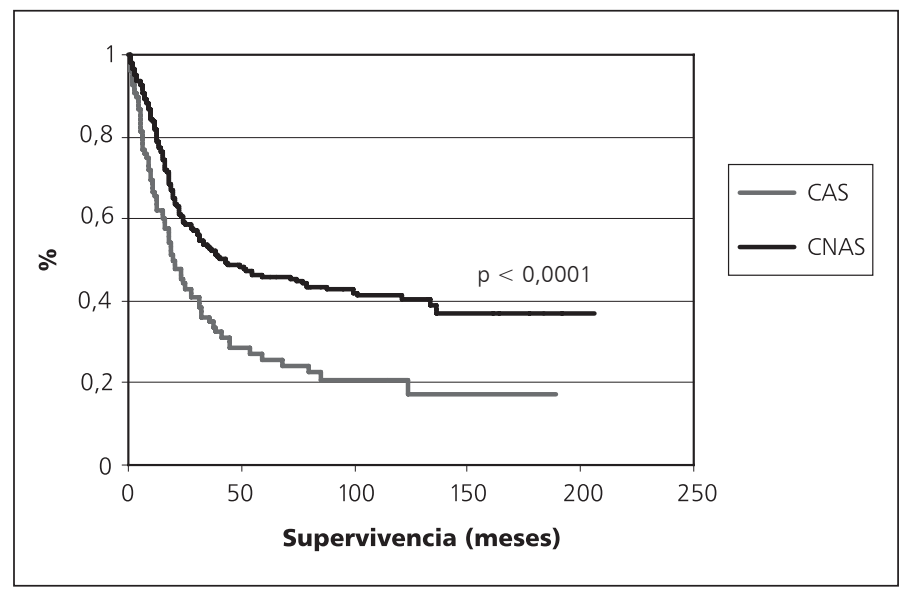

Figura 3. Supervivencia según tipo histológico para tumores gástricos avanzados.
En nuestro estudio, el CAS resultó ser significativamente más frecuente en sujetos jóvenes y en mujeres con una proporción hombre-mujer de 1,2:1 y $2,4: 1$ para CAS y CNAS respectivamente $(\mathrm{p}<0,001)$; la razón de esta asociación no ha sido determinada aunque ciertas hipótesis plantean algún tipo de influencia hormonal, demostrando Matsui et al, con estudio inmunohistoquímico, que CAS son más frecuentemente positivos para receptores de estrógenos que otros tipos histológicos ${ }^{24,25,27,29,31,32}$.

A diferencia de lo publicado por algunos autores respecto a la ubicación cardial más frecuente de las neoplasias gástricas en anillo de sello, en nuestra serie observamos que se localizaron con mayor frecuencia en el 1/3 medio del estómago al igual que lo reportado por Kim y Tejer, junto con ser los CAS lesiones típicamente infiltrativas y difusas tipo linitis $(\mathrm{p}<0,001)^{24,28,30,31}$.

Aún cuando no observamos diferencias estadísticas en el nivel de infiltración (T) entre ambos grupos, el compromiso linfonodal resultó ser significativamente más frecuente en los CAS; en este sentido diversos autores plantean que el peor pronóstico observado en los CAS, más que por el tipo histológico, estaría dado por el mayor compromiso ganglionar linfático que presentan estos pacientes al momento del diagnóstico respecto a los CNAS, sin embargo, trabajos recientes continúan demostrando su desfavorable pronóstico al ser analizado en grupos de tumores que no presentan diferencias estadísticas en cuanto al compromiso ganglionar ${ }^{26,33-36}$.

En nuestra serie, la supervivencia global a 5 años fue $37 \%$ y $51 \%$ para CAS y CNAS respectivamente $(\mathrm{p}=$ 0,02 ); sin embargo, al analizar la supervivencia para el subgrupo de tumores incipientes y avanzados observamos que la supervivencia para tumores incipientes CAS y CNAS fue $95 \%$ y $87 \%$ respectivamente $(p=0,07)$ mientras que para tumores avanzados esta fue de $26 \%$ y $45 \%(p<0,0001)$ para CAS y CNAS respectivamente. Estos datos concuerdan con lo reportado en cuanto al distinto comportamiento pronóstico 
de los CAS observado para tumores incipientes y avanzados, donde si bien en lesiones incipientes no observamos diferencias estadísticas significativas, se aprecia una clara tendencia del mejor pronóstico asociado al CAS y muy por el contrario se asocia a un pronóstico desfavorable en lesiones avanzadas, tal cual lo reportaron Otsuji y Hyung, entre $\operatorname{otros}^{23,24,27,33,34}$.

Nuestros resultados nos permiten concluir el peor pronóstico asociado al tipo histológico CAS respecto a los otros tipos histológicos, información crucial que, aportada al clínico, deberá ser considerada al momento de decidir terapias para perfiles tumorales específicos. Es importante tener en cuenta que la evidencia disponible hoy en día respecto al valor pronóstico independiente del tipo histológico es todavía controversial, permaneciendo todavía el nivel de infiltración tumoral y compromiso ganglionar linfático como los principales factores pronósticos independientes de supervivencia.

Hasta ahora lo más importante continúa siendo pesquisar cada vez más tumores incipientes con el consiguiente mejor pronóstico asociado y por otro lado, trabajar en la identificación de nuevos biomarcadores que permitan reconocer poblaciones con mayor riesgo de desarrollar un CG y, dentro de este grupo, quienes desarrollarán lesiones más agresivas, recurrencia de la enfermedad o refractariedad a los tratamientos complementarios.

\section{Referencias}

1. Medina E, Kaempfer AM. Mortalidad por câncer en Chile: consideraciones epidemiológicas. Rev Med Chile 2001; 129: 1195-1202.

2. http://deis.minsal.cl/ev/mortalidad_causas/cancer_estomago/series/consulta.asp. Ministerio de Salud de Chile.

3. Guías clínicas GES 2006 para Cáncer Gástrico, Ministerio de Salud de Chile.

4. Armijo R. Descriptive epidemiology of cancer in Chile: 1973-78. Nati Cancer Inst Monogr 1982; 62: 91-94.

5. Calderón M, Csendes A, Ospina C. Cambios en la localización tipo histopatológico del cáncer gástrico en el período 1975-2005. Rev Chil Cir 2007; 59: 366-369.

6. Medina E, Kaempffer AM. Mortalidad del adulto en Chile. Rev Med Chile 2000; 128: 1144-1149.

7. Astete G, Lynch O, Madariaga J, Zilic M, Martínez L. Patrón pronóstico de invasión serosa (S2) en carcinoma gástrico. Rev Chil Cir 2002; 54: 644-648.

8. Noguchi Y, Imada T, Matsumoto A. Radical surgery for gastric cancer: a review of the experience. Cancer 1989; 64: 2053-2062.

9. Baba H, Korenaga D, Okamura T, Saito A, Sugimachi K. Prognosis factors in gastric cancer with serosal invasion. Arch Surg 1989; 124: 1061-1064.
10. Al-Moundhri MS, Al-Bahrani B, Burney IA, Nirmala V, Al-Madhani A, Al-Mawaly K, et al. The prognostic determinants of gastric cancer treatment outcome in Omani Arab patients. Oncology 2006; 70: 90-96.

11. Orsenigo E, Carlucci M, Braga M, Tomajer V, Di Palo S, Tamburini A, et al. Prognostic factors of gastric neoplasms: experience with 1,074 cases undergoing surgical treatment at a single center. Suppl Tumori 2005; 4 : S86-87.

12. Alici S, Kaya S, Izmirli M, Tuncer I, Dogan E, Ozbek $\mathrm{H}$, et al. Analysis of survival factors in patients with advanced-stage gastric adenocarcinoma. Med Sci Monit 2006; 12: CR221-229.

13. Ojima H, Araki K, Kato T, Okamura K, Manda R, Hirayama I, et al. Clinicopathological characteristics and outcome indicators of stage II gastric cancer according to the Japanese classification of gastric cancer. Anticancer Res 2006; 26: 1385-1390.

14. Gasparini G, Inelmen EM, Enzi G, Santoro C, Sergi G, Cardin F, et al. Clinical and prognostic aspects of gastric carcinoma in the elderly. J Gastrointest Surg 2006; 10 : 395-401.

15. Bando E, Kojima N, Kawamura T, Takahashi S, Fukushima N, Yonemura Y. Prognostic value of age and sex in early gastric cancer. Br J Surg 2004; 91: 1197-1201.

16. Wang CS, Hsieh CC, Chao TC, Jan YY, Jeng LB, Hwang TL et al. Resectable gastric cancer: operative mortality and survival analysis. Chang Gung Med J 2002; 25: 216-27.

17. Casariego E, Pita S, Rigueiro MT, Pertega S, Rabunal $\mathrm{R}$, Garcia ME, et al. Survival and prognostic factors for gastric cancer. Analysis of 2,334 patients. Med Clin 2001; 117: 361-365.

18. Buonadonna A, Lombardi D, De Paoli A, Bidoli E, Frustaci S. Adenocarcinoma of the stomach: univariate and multivariate analyses of factors associated with survival. Suppl Tumori 2003; 2: S31-34.

19. Green D, Ponce de Leon S, León-Rodríguez E, SosaSánchez R. Adenocarcinoma of the stomach: univariate and multivariate analysis of factors associated with survival. Am J Clin Oncol 2002; 25: 84-89.

20. Hamilton S, Aaltonen L. Tumors of the Stomach. En: Pathology and Genetics of Tumours of the Digestive System. Lyon, France: IARC Press; 2000; 44.

21. Lauren $P$. The two main histological types of gastric carcinoma: Diffuse and so called intestinal-type carcinoma. An attempt at a histo-clinical classification. Acta Pathol Microbiol Scand 1965; 64: 31-49.

22. Ming SC. Gastric carcinoma: A pathobiological classification. Cancer 1977; 39: 2475-2485.

23. Hyung WJ, Noh SH, Lee JH, Huh JJ, Lah KH, Choi $\mathrm{SH}$, et al. Early gastric carcinoma with signet ring cell histology. Cancer 2002; 94: 78-83.

24. Otsuji E, Yamaguchi T, Sawai K, Takahashi T. Characterization of signet ring cell carcinoma of the stomach. J Surg Oncol 1998; 67: 216-220. 
25. Maehara Y, Sakaguchi Y, Moriguchi S, Orita H, Korenaga D, Kohnoe S, et al. Signet ring cell carcinoma of the stomach. Cancer 1992; 69: 1645-1650.

26. Venturelli F, Cárcamo C, Venturelli A, Cárcamo M, Born M, Jara C, y col. Análisis de sobrevida del cáncer gástrico en anillo de sello según profundidad y compromiso linfonodal. Rev Chil Cir 2008; 60: 398-402.

27. Yokota T, Kunii Y, Teshima S, Yamada Y, Saito T, Kikuchi S, et al. Signet ring cell carcinoma of the stomach: a clinicopathological comparison with the other histological types. Tohoku J Exp Med 1998; 186: 121-130.

28. Adachi Y, Yasuda K, Inomata M, Sato K, Shiraishi N, Kitano S. Pathology and prognosis of gastric carcinoma: well versus poorly differentiated type. Cancer 2000; 89: 1418-1424.

29. Antonioli DA, Goldman H. Changes in the location and type of gastric adenocarcinoma. Cancer 1982; 50: 775781.

30. Theuer CP, Nastanski F, Brewster WR, Butler JA, Anton-Culver H. Signet ring cell histology is associated with unique clinical features but does not affect gastric cancer survival. Am Surg 1999; 65: 915-921.

31. Kim DY, Park YK, Joo JK, Ryu SY, Kim YJ, Kim SK, et al. Clinicopathological characteristics of signet ring cell carcinoma of the stomach. ANZ J Surg 2004; 74: 1060-1064.

32. Matsui M, Kojima O, Kawakami S, Uehara Y, Takahashi $\mathrm{T}$. The prognosis of patients with gastric cancer possessing sex hormone receptors. Surg Today 1992; 22: 421-425.

33. Piessen G, Messager M, Leteurtre E, Jean-Pierre T, Mariette C. Signet ring cell histology is an independent predictor of poor prognosis in gastric adenocarcinoma regardless of tumoral clinical presentation. Ann Surg 2009; 250: 878-887.

34. Li C, Kim S, Lai JF, Hyung WJ, Choi WH, Choi SH, et al. Advanced gastric carcinoma with signet ring cell histology. Oncology 2007; 72: 64-68.

35. Kunisaki C, Shimada H, Nomura M, Matsuda G, Otsuka $\mathrm{Y}$, Akiyama $\mathrm{H}$. Therapeutic strategy for signet ring cell carcinoma of the stomach. Br J Surg 2004; 91: 13191324.

36. Kim DY, Seo KW, Joo JK, Park YK, Ryu SY, Kim HR, et al. Factors in patients with node-negative gastric carcinoma: a comparison with node-positive gastric carcinoma. World J Gastroenterol 2006; 12: 1182-1186. 